\title{
SAMU versus Trauma Center
}

\author{
Ph. Vichard
}

Service d'Orthopédie-Traumatologie, CHRU Besançon, F-25030 Besançon Cedex

Dans un journal consacré en grande partie à la Traumatologie et qui se veut européen, il est logique de s'intéresser à la relève et surtout l'accueil des blessés dans les Centres Hospitaliers français.

Des comparaisons doivent être faites entre les politiques sanitaires des états européens ou très industrialisés. L'Europe, c'est non seulement le libre échange des marchandises, mais aussi celui des idées.

Il paraîtra peut-être saugrenu d'opposer (au plan des idées) des institutions qui n'ont pas le même but. Cette confusion, on le verra, n'est qu'apparente...

Le sigle SAMU inventé en France évoque la médicalisation de la relève des blessés prise en mains par les Pouvoirs publics. C'est une chaîne de dévouements et de compétences qui aboutit au soulagement de détresses. Cette institution ne devrait pas être suspectée... Ce n'est pas mon intention, mais je souhaite seulement en contester la structure et la ramener à sa mission primitive : le traitement des situations critiques.

En effet, le monopole de la prise en charge des blessés et des urgences en général par les Pouvoirs publics s'étend maintenant à presque tout le territoire national et il soulage non seulement les détresses physiques sur le terrain, mais en vient dans certains centres à prendre en charge, au pavillon d'accueil des Centres Hospitaliers dont il peut avoir la charge, toutes les inadaptations.

TRAUMA CENTER : cette appellation américaine a fait irruption dans les réunions scientifiques françaises, ce qui nous a fait découvrir brusquement que
l'Amérique empirique et privatiseuse avait pris en mains l'accueil et le traitement des traumatisés, ce qui, en France tout au moins, souffre gravement de l'absence de Centres de Traumatologie, de la dérive des pavillons d'accueil où on reçoit tout, trie tout. Comme si le sapeur-pompier, le policier, voire le passant, était incapable de faire le diagnostic du traumatisme, lequel devrait conduire les blessés vers le Centre de Traumatologie exactement comme n'importe quel « laïc » conduit une parturiente à la maternité, sans le truchement de véritables parkings, toujours surchargés car polyvalents, où s'entassent sans espoir d'une progression rapide les intoxiqués, les drogués, les abdomens aigus, les immigrés en quête d'un toit, ou même ceux qui ont simplement le mal de vivre...

Le tri n'est pas cela. Dans le cas de notre spécialité il s'adresse à des traumatisés hospitalisés ou examinés dans une formation qui leur est exclusivement destinée et qui en France n'existe pas. C'est le choix des lésions à traiter en premier, réalisé par des Chirurgiens cultivés, rompus à la traumatologie générale et non par des généralistes vacataires, ou des étudiants en médecine.

Après ces quelques mots, on comprendra mieux la comparaison, un peu inattendue, à propos des SAMU, fruits de conceptions hexagonales visant à faire prendre en surface toutes les urgences dont les traumatiques, sur tout le territoire, et ensuite à les répartir à l'intérieur des Centres Hospitaliers.

Certains enthousiastes du système actuel célèbrent la porte unique derrière laquelle tous les médecins hospitaliers attendent l'urgence. Cette attente vigilante et de tous les instants est un mythe. Compte tenu de la diversité des cas cliniques envisagés, la sentinelle postée à l'entrée de l'hôpital est très souvent incompétente.

Il vaudrait mieux faire sauter ce goulot d'étranglement, réputé économique, efficace, pour répartir grâce au bon sens les urgences vers les services ou groupes de services (le Centre de Traumatologie en est un), destinés à terme à les traiter. Quelques modifications architecturales, une réforme des mentalités (plus de sens et de préoccupations cliniques, moins d'égoïsme des médecins quant à leur pôle d'intérêt) assureraient le progrès technique, comme l'accélération de la prise en charge. Il $\mathrm{y}$ a un moment où la concentration de nombreux malades, à l'accueil, réclamée par l'administration au nom du rendement des équipements, et des personnels devient onéreuse. Les services d'accueil toujours saturés, sans cesse renforcés, deviennent petit à petit des gouffres à crédit. Ils se réservent des équipements, par exemple des blocs opératoires dits d'urgence qui font double emploi avec ceux réservés aux malades programmés. Or, le blessé pourrait parfaitement être opéré dans le bloc général moyennant une révision de ses moyens d'accès!

Cette politique ambitieuse, qui consiste à relever toutes les urgences puis à les trier parfois à l'aide d'examens complémentaires sophistiqués, sera toujours en perpétuel devenir. Elle a multiplié... elle multipliera sans fin 
les personnels et les matériels. Comme, nous dit-on, les crédits de santé ne peuvent croître indéfiniment vis-à-vis du «PNB », il faudra faire des choix, restreindre les équipements lourds, donc retarder la création des Centres de Traumatologie, nier l'évidence, pour conserver des structures polyvalentes où le travail ne pourra jamais être réalisé en profondeur.

$\mathrm{Au}$ total, il faut dans les CHU ou CHR au moins « renverser la vapeur ", diversifier les points d'accueil des urgences, les rapprocher du praticien adéquat. Chaque spécialité a ses urgences. Elle doit y consacrer ses propres locaux et son propre personnel.

Il faut par ailleurs trancher entre le jacobinisme sanitaire d'une part et la sélection par les décideurs régionaux de formations spécialisées (Centres de Secours entre autres...) dont le besoin se fait sentir, ne pas se laisser imposer un schéma national ambitieux de moyens de relève et d'accueil qui soit financé indûment par les Centres Hospitaliers eux-mêmes. Sinon, la Santé rejoindra l'Education Nationale qui gère tout, donc gère mal, et qui, un siècle après Jules Ferry, découvre... l'existence d'une proportion inattendue d'illétrés.
Que nos amis des SAMU, avec lesquels nous collaborons, ne se sentent pas offensés. Ce qui compte, dans la relève des blessés, c'est moins l'origine des ambulances, que la conscience et la technique des médecins qui les montent. Bien plus, les praticiens des SAMU se verraient volontiers débarrassés des fausses urgences pour se consacrer exclusivement aux vraies détresses... D'autres pays industrialisés ont sans doute adopté une organisation plus judicieuse que la nôtre. Ils disposent, eux, de Centres de Traumatologie. De grâce..., qu'on en crée en France! 